\title{
Spatial variation of a giant spin-orbit effect induces electron confinement in graphene on $\mathrm{Pb}$ islands
}

\author{
Fabian Calleja ${ }^{1}$, Héctor Ochoa ${ }^{2}$, Manuela Garnica ${ }^{1,3}$, Sara Barja ${ }^{1,3}$, Juan Jesús Navarro ${ }^{1}$ \\ Andrés Black ${ }^{1,3}$, Mikhail M. Otrokov ${ }^{4,5}$, Evgueni V. Chulkov ${ }^{4,6}$, Andrés Arnau ${ }^{4,6}$, \\ Amadeo L. Vázquez de Parga ${ }^{1,3}$, Francisco Guinea ${ }^{2 \star}$ and Rodolfo Miranda ${ }^{1,3 \star}$
}

\begin{abstract}
The electronic band structure of a material can acquire interesting topological properties in the presence of a magnetic field or as a result of the spin-orbit coupling ${ }^{1-3}$. We study graphene on Ir, with $\mathrm{Pb}$ monolayer islands intercalated between the graphene sheet and the Ir surface. Although the graphene layer is structurally unaffected by the presence of the $\mathrm{Pb}$ islands, its electronic properties change markedly, with regularly spaced resonances appearing. We interpret these resonances as the effect of a strong and spatially modulated spin-orbit coupling, induced in graphene by the $\mathrm{Pb}$ monolayer. As well as confined electronic states, the electronic spectrum has a series of gaps with non-trivial topological properties, resembling a realization of the quantum spin Hall effect proposed by Bernevig and Zhang ${ }^{4}$.
\end{abstract}

The use of graphene in spintronic devices has been suggested on the basis of the very large values of the spin diffusion length expected for graphene ${ }^{5}$. Apart from this role as spin 'conserver', it would be highly desirable to be able to manipulate the spins in graphene, which could be achieved by enhancing the rather weak spin-orbit (S-O) coupling characteristic of the $\mathrm{C}$ atoms. Enhancing the $\mathrm{S}-\mathrm{O}$ interaction in graphene also has implications from a rather fundamental point of view. The existence of non-trivial topological properties induced by a suitable $\mathrm{S}-\mathrm{O}$ coupling (the topological insulator state) was first proposed for two-dimensional graphene $e^{6,7}$. Considering the negligible value of the $\mathrm{S}-\mathrm{O}$ coupling in graphene, the observation of topological insulator features in graphene, however, would require extremely low temperatures and a high degree of perfection ${ }^{8}$. Alternative routes to modify the S-O interaction in graphene have been put forward ${ }^{9}$. Although these proposals have not yet been realized, the internal degrees of freedom of graphene allow pseudo-magnetic fields ${ }^{10}$ induced by lattice deformations. The existence of these pseudo-fields leads to the possibility of confined electronic states in graphene, which has been experimentally reported around nanobubbles ${ }^{11}$ and wrinkles ${ }^{12}$. Here we show that a spatial variation in the intensity of the S-O coupling in graphene induced by the partial intercalation of heavy atoms, such as $\mathrm{Pb}$, with the appropriate symmetries generates a pseudomagnetic field that confines electrons and results in a series of sharp, Landau-like levels in the density of states of graphene.
Graphene can be grown on $\operatorname{Ir}(111)$ by direct decomposition of $8 \times 10^{-8}$ torr of ethylene at $1,400 \mathrm{~K}$ on the Ir surface ${ }^{13,14}$. Largescale images show the presence of an almost complete monolayer of graphene, with some wrinkles (see also Supplementary Fig. 1a). The bonding between graphene and $\operatorname{Ir}(111)$ is weak $\mathrm{k}^{15,16}$. Most of the graphene overlayer presents a well-known incommensurate $9.3 \times 9.3$ moiré superstructure ${ }^{13}$ with a geometrical corrugation of $35 \mathrm{pm}$ (ref. 16), arising from the lattice mismatch of graphene with respect to the Ir substrate. In addition, the graphene overlayer is weakly p-doped by charge transfer to the substrate, with the Dirac energy at $+100 \mathrm{meV}$ (above the Fermi level), and it exhibits the conical band dispersion characteristic of free-standing graphene ${ }^{17}$.

Intercalation of $\mathrm{Pb}$ under the graphene monolayer is easily achieved by evaporating $\mathrm{Pb}$ onto the graphene/ $\operatorname{Ir}(111)$ (for short, $\mathrm{gr} / \mathrm{Ir}(111)$ ) sample kept at $800 \mathrm{~K}$ if the graphene monolayer presents domain boundaries, wrinkles or other extended defects ${ }^{18,19}$ (see Supplementary Information A.1 for details). Figure 1a shows a representative, large-scale scanning tunnelling microscopy (STM) image of the partly $\mathrm{Pb}$-intercalated graphene, corresponding to an intercalation ratio of the order of $20 \%$ of the surface (see also Supplementary Fig. 1b). Some $\mathrm{Pb}$-intercalated islands appear in the terraces (frequently close to graphene wrinkles), but most of the intercalated regions are located at the step edges. The $\mathrm{Pb}$ coverage has been selected so that the lateral size of the islands ranges from 4 to $12 \mathrm{~nm}$ (average of $6 \mathrm{~nm}$ ) and their average separation is around $35 \mathrm{~nm}$.

Figure $1 \mathrm{~b}$ shows an atomically resolved STM image acquired at the edge of one of the $\mathrm{Pb}$-intercalated islands. The right part of the image corresponds to the gr/Ir region, where two hexagonal lattices can be seen: the atomic lattice of graphene and the $9.3 \times 9.3$ moiré structure. As well as these two lattices, the intercalated region on the left exhibits an additional rectangular structure arising from the $\mathrm{Pb}$ interlayer. Graphene's atomic lattice covers both regions continuously across the atomic step, as shown by the line profile in Fig. 1c, which also demonstrates that graphene on top of the $\mathrm{Pb}$-intercalated islands presents a negligible corrugation, certainly well below the small corrugation of $\operatorname{gr} / \operatorname{Ir}(111)$. The apparent height of the islands (see Fig. 1c and histogram in Supplementary Fig. 1b) indicates that the intercalated layer is just one $\mathrm{Pb}$ atom thick.

${ }^{1}$ Instituto Madrileño de Estudios Avanzados en Nanociencia, Cantoblanco 28049, Madrid, Spain. ${ }^{2}$ Instituto de Ciencia de Materiales de Madrid, Consejo Superior de Investigaciones Científicas, Cantoblanco 28049, Madrid, Spain. ${ }^{3}$ Departamento de Física de la Materia Condensada and IFIMAC, Universidad Autónoma de Madrid, Cantoblanco 28049, Madrid, Spain. ${ }^{4}$ Donostia International Physics Centre (DIPC), Paseo Manuel de Lardizabal 4, San Sebastian 20018, Spain. ${ }^{5}$ Tomsk State University, Tomsk 634050, Russia. ${ }^{6}$ Departamento de Física de Materiales UPV/EHU and Centro de Física de Materiales (CFM), Centro Mixto CSIC-UPV/EHU, Paseo Manuel de Lardizabal 5, San Sebastian 20018, Spain. *e-mail: paco.guinea@icmm.csic.es; 
a

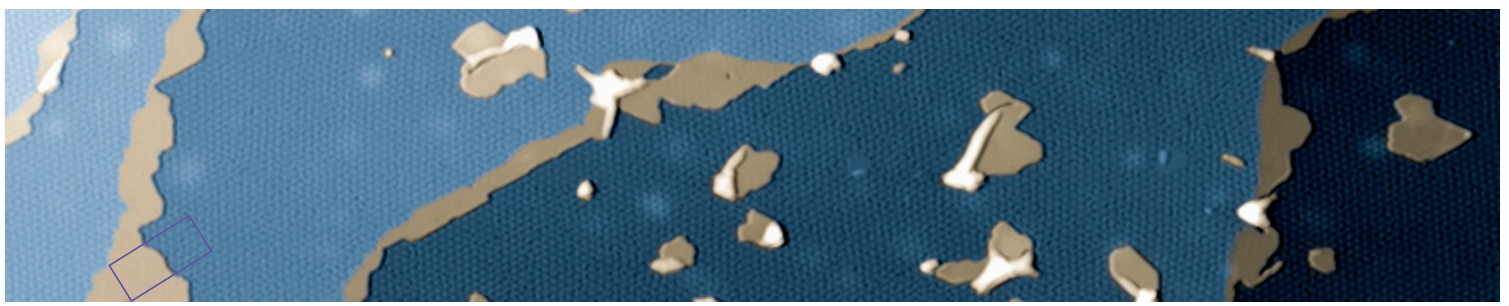

b
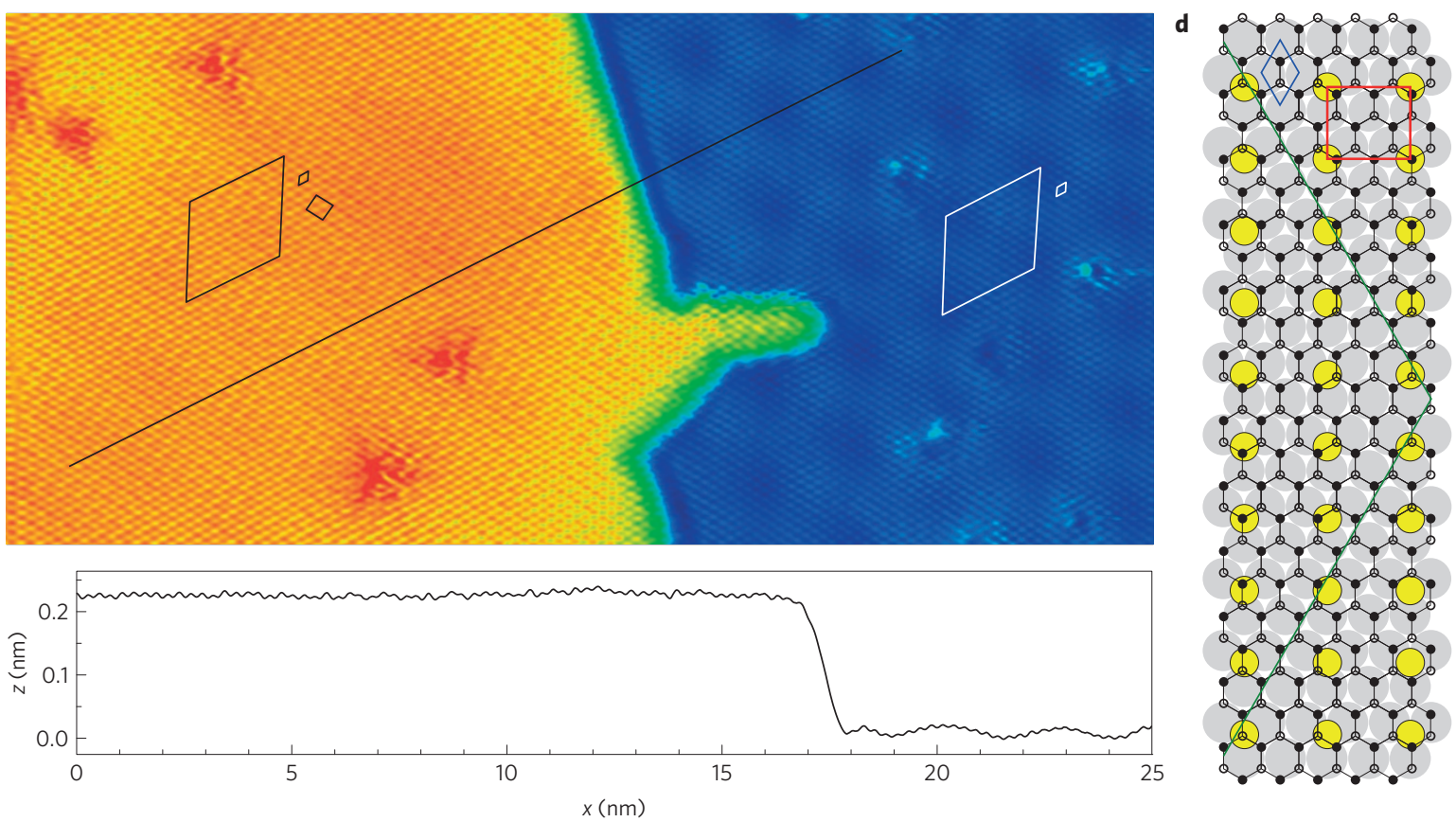

Figure 1 | Structure of Pb-intercalated graphene. a, Large-scale STM image $\left(470 \times 90 \mathrm{~nm}^{2}\right)$ of gr/Ir(111) after deposition of Pb at $800 \mathrm{~K}$. The image has been taken at $80 \mathrm{~K}$. There are areas intercalated with $\mathrm{Pb}$ close to the steps and intercalated islands in the middle of the terraces, often associated with the presence of wrinkles (white areas) in pristine gr/Ir(111). The intercalated areas are indicated by the beige shaded regions, as opposed to the blue tint given to the non-intercalated regions, for an easier identification. b. Atomically resolved STM image $\left(28 \times 13 \mathrm{~nm}^{2}\right)$ acquired across the border between $\mathrm{gr} / \mathrm{Pb} / \mathrm{Ir}(111)$ (left) and gr/Ir(111) (right) in a region similar to the one marked by the purple rectangle in $\mathbf{a}$, taken at $4.6 \mathrm{~K}$. The relevant unit cells on the intercalated and non-intercalated regions are highlighted in black and white, respectively. c, Height profile along the black line in the image shown in $\mathbf{b}$ d, Schematic model of the atomic arrangement obtained by STM and LEED: grey and yellow circles represent Ir and Pb atoms respectively. Black filled and open circles represent the two graphene sublattices. The unit cells of moiré, Pb-intercalated layer and graphene are shown in green (half of the unit cell), red and blue respectively. Notice that the $\mathrm{Pb}$ and $\operatorname{Ir}(111)$ lattices are commensurate.

Figure 1d shows the atomic structure of the intercalated system in real space as deduced from low-energy electron diffraction (LEED) patterns and Fourier transforms of large-scale, atomically resolved STM images (see Supplementary Information A.2). According to our measurements, neither the graphene nor the Ir lattice parameters change upon $\mathrm{Pb}$ intercalation, so that the moiré pattern is maintained (shown in green). Intercalated $\mathrm{Pb}$ atoms form a rectangular lattice (shown in red) which corresponds to a $c(4 \times 2)$ superstructure commensurate with $\mathrm{Ir}$ and, therefore, incommensurate with graphene. The Dirac point for the $\mathrm{Pb}$ intercalated graphene is estimated to be at $\pm 110 \pm 20 \mathrm{meV}$ from the diameter of the inter-valley scattering half-moon rings observed in the STM Fourier transforms (see Supplementary Information A.2.3 and Supplementary Fig. 3b). Notice that the density of $\mathrm{Pb}$ atoms in the intercalated layer is rather high-one seventh of the density of $\mathrm{C}$ atoms in graphene.

The local density of states (LDOS) at the graphene regions intercalated with $\mathrm{Pb}$ was measured by scanning tunnelling spectroscopy (STS) at $4.6 \mathrm{~K}$ using standard lock-in techniques (see Supplementary Information A.3). Figure 2 shows a representative differential conductance spectrum measured on the 10 -nm-wide $\mathrm{Pb}$-intercalated island that appears in the inset of Fig. 2. Up to eight clearly defined, intense and sharp peaks in an $\approx 3 \mathrm{eV}$ wide region are observed. The full-width at half-maximum of the peaks is of the order of $40 \mathrm{meV}$. The (rather featureless) spectra recorded on the pristine $\mathrm{gr} / \mathrm{Ir}(111)$ surface or on a completely $\mathrm{Pb}$-intercalated graphene monolayer do not show this series of sharp resonances, as demonstrated in Supplementary Fig. 5 (Supplementary Information A.3). By placing the estimated Dirac point at $-110 \mathrm{meV}$ below the Fermi energy (orange line in Fig. 2; that is, the Pb-intercalated graphene is slightly $\mathrm{n}$-doped), the peaks seem to be symmetrically distributed above and below it, with the $n=0$ peak missing. The separation between peaks is nearly constant (that is, $E_{n} \sim n$ ), at an average of $340 \mathrm{meV}$ for an island of this size. The effect is observed in all $\mathrm{gr} / \mathrm{Pb}$ islands, and it is robust, independent of the detailed shape of the $\mathrm{Pb}$ islands and of the defects present at the edges (or interior) of the islands. The peak separation is roughly inversely proportional to the size of the islands and the peaks can be clearly detected even at $80 \mathrm{~K}$ (see Supplementary Information A.3 for details). These quantized energy levels are similar to the Landau levels that arise when electrons in two-dimensional systems are confined by an external magnetic field.

As shown in Fig. 3, the STS spectra recorded when going from the $\mathrm{Pb}$-intercalated graphene region (red cross on a and red curve 


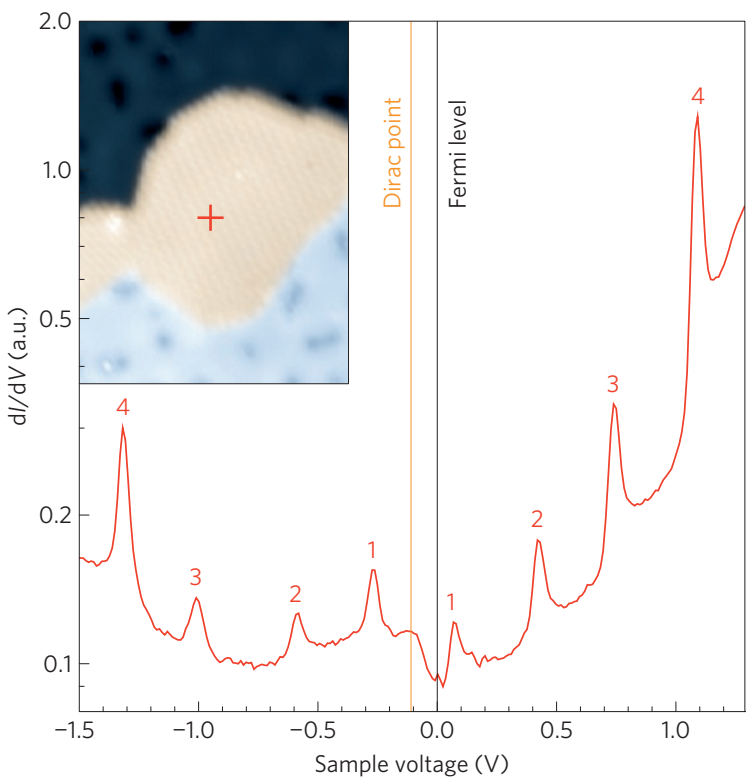

Figure 2 | STS in a Pb-intercalated graphene island. Differential conductance spectrum recorded at $4.6 \mathrm{~K}$ on the centre of the $10-\mathrm{nm}$-wide $\mathrm{gr} / \mathrm{Pb} / \mathrm{Ir}(111)$ island shown in the inset. The tunnelling gap was stabilized at $-1.5 \mathrm{~V}$ and $50 \mathrm{pA}$. The orange line marks the Dirac point obtained from STM-Fast Fourier transform analysis and the red numbers indicate the assigned quantum numbers. a.u., arbitrary units.

on c) into the gr/Ir area (blue cross on a and blue curve on c) demonstrate that all the peaks shift, essentially in a rigid fashion on entering the gr/Ir region. The quantized levels (labelled from 1 to 6 , starting at the Dirac point) appear in both regions with the same energy separation $(\sim 340 \mathrm{meV})$, but shifted in energy by nearly $200 \mathrm{meV}$, which is almost exactly the difference in Dirac energy between both regions $(-110 \mathrm{meV}$ for $\mathrm{gr} / \mathrm{Pb}$ and $+100 \mathrm{meV}$ for $\mathrm{gr} / \mathrm{Ir}$ ). The smooth shift of the quantized levels can be clearly seen in the central part of Fig. 3c, where we show (in inverted grey scale) the spatial map of the $\mathrm{d} I / \mathrm{d} V$ intensity along the green arrow in Fig. 3a. The shift follows strictly the variation of the Dirac point when moving between the two regions (in fact there is an exception, namely the unlabelled peak between peaks 5 and 6 , which shifts by nearly $300 \mathrm{meV}$ and coalesces with $n=5$ well into the $\mathrm{gr} / \mathrm{Ir}$ region). All the peaks are clearly visible even $10 \mathrm{~nm}$ away from the physical edge of the $\mathrm{Pb}$-intercalated graphene region into the gr/Ir region (that is, at the position signalled by the blue cross).

Figure $3 \mathrm{~d}$ shows the energy position of the quantized levels as a function of their quantum number, $n$. A linear dependence that intersects at the respective Dirac points is evident for both $\mathrm{gr} / \mathrm{Pb} / \operatorname{Ir}(111)$ and $\operatorname{gr} / \operatorname{Ir}(111)$ data sets. Under an actual magnetic field, the energy of the Landau levels, $E_{\mathrm{n}}$, is expected to depend linearly on $n\left(E_{\mathrm{n}}=\hbar \omega_{\mathrm{c}}(n+1 / 2)\right)$ for a two-dimensional electronic system with parabolic dispersion, on $n^{1 / 2}$ for graphene $e^{20}$ $\left(E_{n}=\operatorname{sgn}(n) \hbar \omega_{c} n^{1 / 2}\right)$ and on $[n(n+1)]^{1 / 2}$ (which is roughly similar to $n$ ) for bilayer graphene, a system with a small gap.

The observation of the sharp resonances described above implies the existence of quasi-localized electronic states. The scale of the confinement, $l$, can be inferred from the gaps, $\Delta$, between resonances, $l \approx v_{\mathrm{F}} / \Delta \approx 2-3 \mathrm{~nm}$. The confinement of electrons in graphene by scalar potential barriers (for example, at the island edges) over lengths much greater than the interatomic distance, $a \approx 0.14 \mathrm{~nm}$, is prevented by the Klein tunnelling associated with the chirality of the wavefunctions ${ }^{21}$, and localized states are typically found only in the presence of defects at the Dirac energy. Furthermore, although the $\mathrm{n}-\mathrm{p}$ barriers $(\approx 200 \mathrm{meV})$ at the interfaces between $\mathrm{gr} / \mathrm{Pb} / \operatorname{Ir}(111)$ and $\mathrm{gr} / \operatorname{Ir}(111)$ could confine electrons, it is hard to imagine that the confinement can extend to states that have energies of several $\mathrm{eV}$, as observed here.

The most common sources of confinement at arbitrary energies are gauge fields $s^{8,10,22}$. The effective magnetic field required to generate the observed gaps is $B(\mathrm{~T}) \approx(25 / l(\mathrm{~nm}))^{2} \approx 80-100 \mathrm{~T}$. The formation of localized levels similar to the Landau levels by an effective magnetic field associated with strains has been reported for nanobubbles in $\mathrm{gr} / \mathrm{Pt}(111)$ (ref. 11) and highly strained wrinkles in twisted bilayers in $\mathrm{gr} / \mathrm{Rh}(111)$ (ref. 12). However, the graphene layer studied here shows no appreciable strains. If we assume that the observed confinement is induced by a varying strain which changes by $\Delta \bar{u}$ over a distance of the order of the size of the $\mathrm{Pb}$ island, $R \approx 19 \mathrm{~nm}$, we obtain $\Delta \bar{u} \approx(a R) /\left(\beta l^{2}\right) \approx 0.05-0.2$, where $\beta=(a / t) \partial t / \partial a \approx 2$ and $t \approx 3 \mathrm{eV}$ is the hopping between nearest neighbour atoms. The graphene layer studied here is flat and uniform, and there is no hint of strain, such as that estimated above.

The linear dispersion of the graphene bands allows the existence of a variety of mechanisms which can induce gauge fields $\mathrm{s}^{22-25}$, other than strains. Hopping between two graphene layers, for instance, can be formulated as a non-Abelian gauge field ${ }^{25}$, and this approach can be generalized in a straightforward way to the Rashba S-O coupling in graphene. In the following, we show that the existence of a strong and spatially non-uniform S-O coupling is consistent with the existence of sharp resonances observed experimentally. The gauge field associated with the $\mathrm{S}-\mathrm{O}$ coupling is more complex than the Abelian field induced by strains, leading to novel structures not observable in real magnetic fields or in the presence of strains.

We have performed density functional theory calculations for ordered arrays of $\mathrm{Pb}$ atoms adsorbed on isolated graphene (see Supplementary Information B.1 for details) that demonstrate that electrons from graphene tunnelling through the $\mathrm{Pb}$ atoms can feel the large S-O interaction characteristic of these heavy atoms (that is, the $\mathrm{S}-\mathrm{O}$ parameter for bulk $\mathrm{Pb}$ is $0.91 \mathrm{eV}$; ref. 26) in such a way that the graphene bands are spin split by a giant S-O coupling larger than $100 \mathrm{meV}$ (see Supplementary Figs 7, 8 and 10). Periodically arranged heavy adatoms have been predicted ${ }^{9}$ to stabilize a Haldane-Kane-Mele phase in graphene $e^{6,7}$. According to the lateral position of the $\mathrm{Pb}$ atoms deduced from LEED measurements, graphene on $\mathrm{Pb} / \mathrm{Ir}(111)$ feels a rectangular $c(4 \times 2)$ superlattice. In this situation, it can be shown (see Supplementary Information B. 2 for details) that graphene $\pi$ electrons are perturbed by spindependent superlattice potentials in such a way that the effective Dirac-like Hamiltonian valid at both inequivalent valleys reads

$$
H=v_{\mathrm{F}} \boldsymbol{\Sigma} \cdot(-i \nabla+\mathbf{A}) \pm A_{0} S_{y}
$$

where $\boldsymbol{\Sigma}=\left( \pm \sigma_{x}, \sigma_{y}\right)$ and $\mathbf{A}=\left(A_{x} S_{y}, A_{y} S_{x}\right)$. Here $\sigma_{i}\left(S_{i}\right)$ are Pauli matrices acting on sublattice (spin) degrees of freedom, and \pm holds for $\pm \mathbf{K}$ valleys.

The field $\mathbf{A}$ can be interpreted as a non-Abelian gauge potential, whereas $A_{0}$ is a scalar potential with opposite sign at each valley and coupled to the spin operator along the armchair direction, which displaces the sequence of Landau levels from zero in opposite directions. This situation is formally similar to the case of twisted graphene bilayers ${ }^{25}$, but with the layer degree of freedom replaced by the spin. As in that case, a non-uniform spatial change of the strength of the $\mathrm{S}-\mathrm{O}$ interaction generated by going from the $\mathrm{Pb}$-intercalated graphene to graphene directly grown on $\mathrm{Ir}$ leads to a spatial modulation of the non-Abelian gauge field, which, in turn, leads to a pseudo-magnetic field confining Dirac electrons. In spite of the local character of S-O coupling, the effect is detected at long distances from the physical edge of the $\mathrm{Pb}$-intercalated islands. This reveals the extraordinarily large distances to which perturbations extend in graphene. We argue that the peaks present at the STS spectra are, thus, due to electronic confinement induced by spatially varying $\mathrm{S}-\mathrm{O}$ fields. 

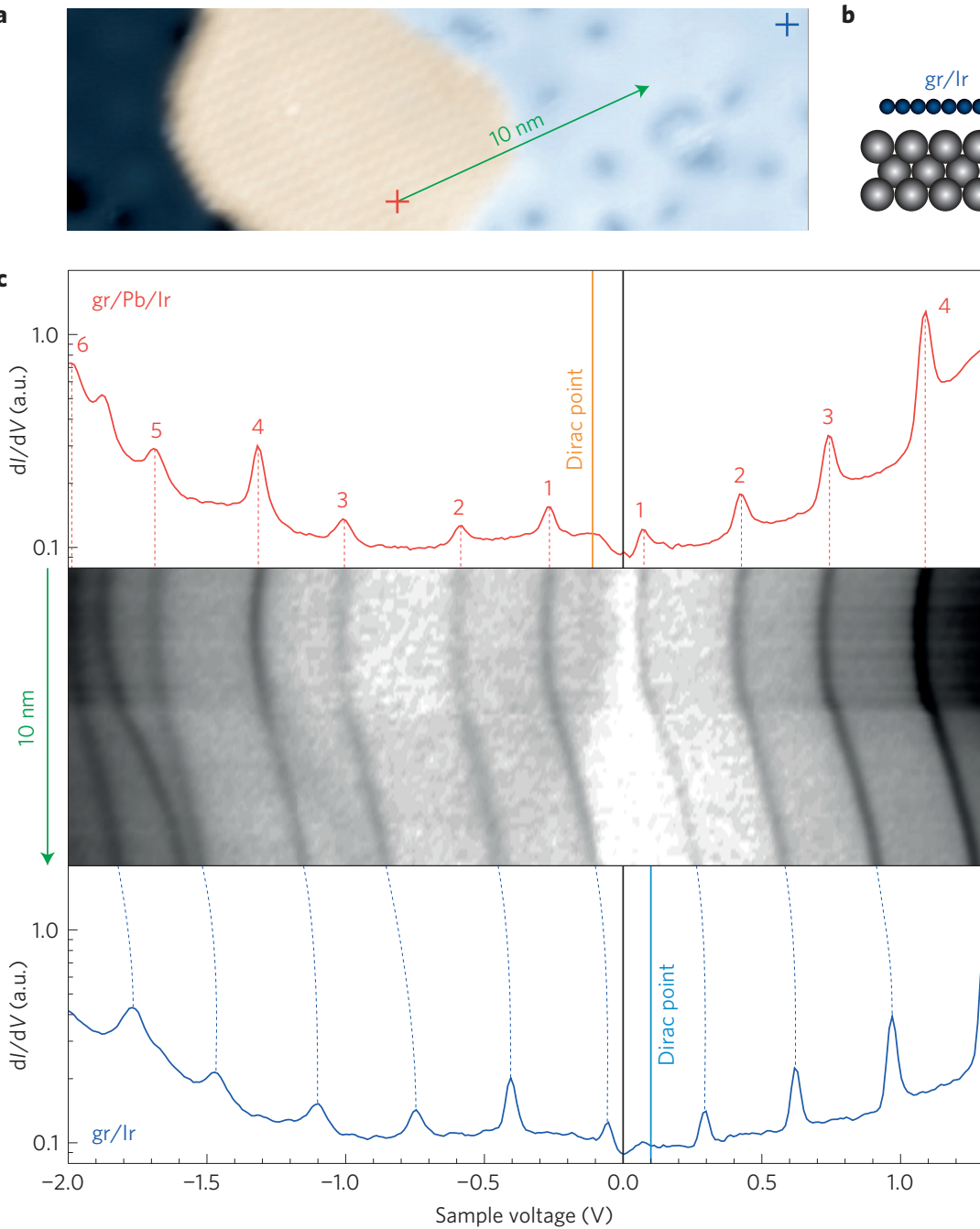

b

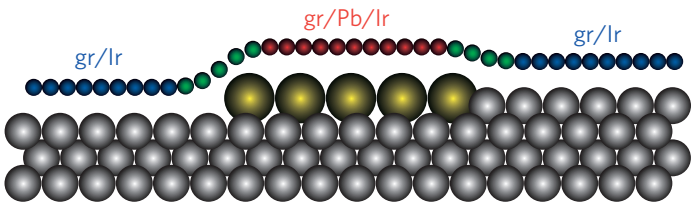

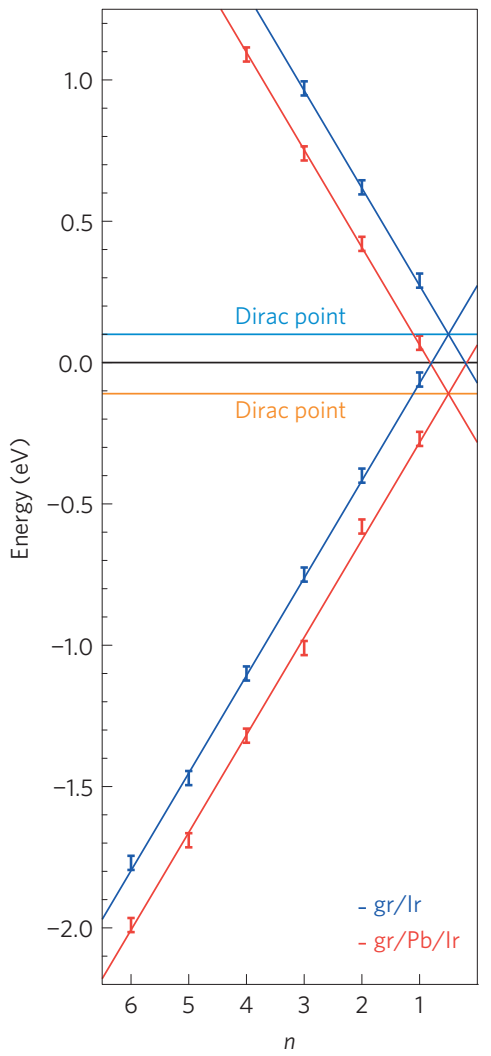

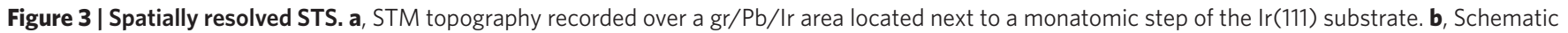

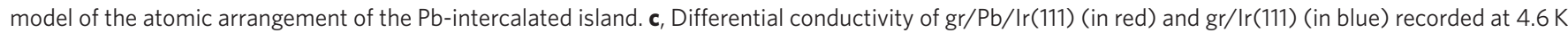
at the points indicated by the crosses in $\mathbf{a}$. The $d / / d V$ intensity map recorded along the green line in $\mathbf{a}$ is shown between the spectra at the extremes. The tunnelling gap was stabilized at $-1.5 \mathrm{~V}$ and $50 \mathrm{pA}$. In all cases, the lock-in signal is plotted on a logarithmic scale for an easier visualization of the peaks. a.u., arbitrary units. d, Energy positions of the peaks as a function of the quantum number. The error bars correspond to the full-width at half-maximum of the corresponding peaks that appear in $\mathbf{c}$.

Figure 4a reproduces schematically the spatial evolution of the intensity of the $\mathrm{S}-\mathrm{O}$ coupling when moving away from the $\mathrm{Pb}$ intercalated region of graphene. Figure $4 \mathrm{~b}$ shows the calculated LDOS within a tight-binding model, which reproduces the previous Hamiltonian at Dirac points and is parameterized to produce electronics bands in agreement with the density functional theory calculations (see Supplementary Information B.2 and B.3 for details). The best agreement between theory and experiment is achieved when a purely Abelian field (either $A_{x}=0$ or $A_{y}=0$ ) is generated, but the sharp peaks survive even in the non-Abelian case. The peaks may be interpreted as pseudo-Landau levels associated with effective magnetic fields with opposite sign for each in-plane spin polarization along the armchair direction. The scalar field displaces the Landau spectra associated with each valley. The system resembles a realization of the quantum spin Hall effect proposed by Bernevig and Zhang ${ }^{4}$. At the edges of the regions where the $\mathrm{S}-\mathrm{O}$ coupling varies we expect in-plane spin-polarized counterpropagating modes, as shown in Fig. 4a. For an odd number of occupied Landau levels at least one Kramers pair of these edge modes is topologically protected against disorder by time-reversal symmetry. The characteristic decay length of these modes into the region where the $\mathrm{S}-\mathrm{O}$ changes (the region changing colour in Fig. 4 a) can be estimated from the experiments in $\approx 20 a$, which is much less than the characteristic length $(\approx 60 a)$ over which the S-O orbit changes in the simulations. The separation between peaks in the calculation roughly follows the expected behaviour for uniform magnetic fields, $E_{n} \propto \sqrt{ } n$, although the inclusion of all the possible $\mathrm{S}-\mathrm{O}$ terms leads to deviations from this sequence. Note that the model includes only the S-O coupling terms in graphene induced by $\mathrm{Pb}$. The nearly constant separation between peaks observed in the experiment may be recovered in a more accurate calculation if more terms (not strictly S-O coupling terms) are included in the model, for instance, opening a spectral gap due to the presence of $\mathrm{Pb}$, as in other graphene superlattices ${ }^{27}$.

Hybrid structures made up of different two-dimensional layers can have properties different from each of their components ${ }^{27,28}$. The combination of a graphene and $\mathrm{a} \mathrm{Pb}$ underlayer leads to a two-dimensional system with electronic resonances not present in either material, associated with the enhancement and the spatial variation of the $\mathrm{S}-\mathrm{O}$ coupling in the graphene layer. To observe the effect, the intercalated atoms would have to generate strong $\mathrm{S}-\mathrm{O}$ coupling in the graphene bands, preserve the sublattice symmetry 

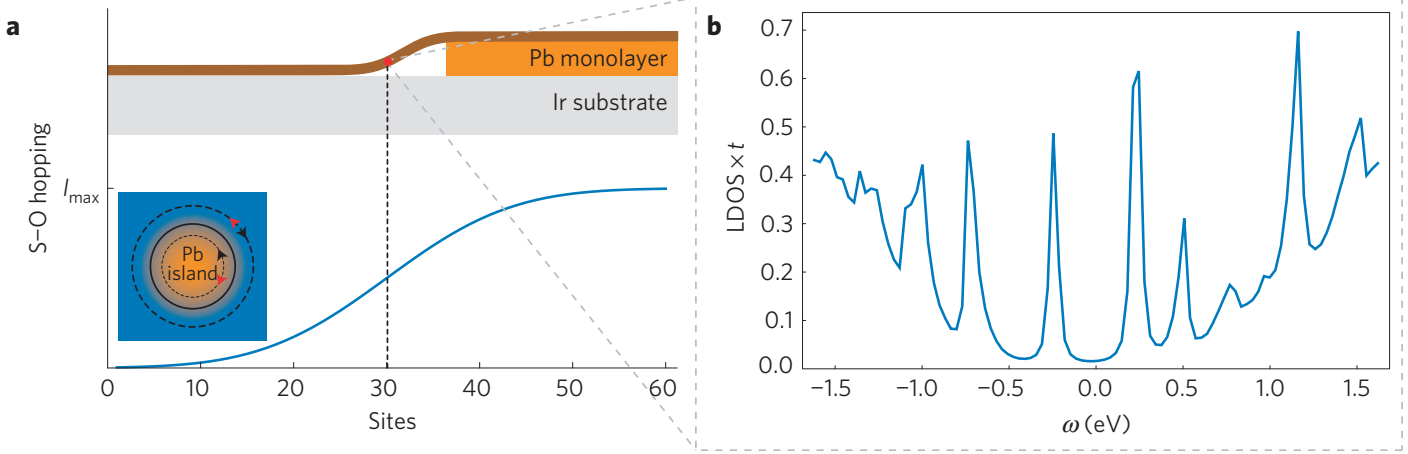

Figure 4 | Spatial variation of the spin-orbit coupling and calculated local density of states. a, Spatial evolution of the S-O coupling across the border of the $\mathrm{Pb}$-intercalated regions. The non-uniform S-O profile follows an error function spread over 60 unit cells of graphene along the armchair direction. The inset shows schematically a $\mathrm{Pb}$ island with its physical edge in black, and the in-plane spin-polarized counter-propagating modes expected at the edges of the region where the $\mathrm{S}-\mathrm{O}$ changes between $\mathrm{Pb}$ islands. The colour of the arrows indicates opposite in-plane spin polarizations. $\mathbf{b}$, LDOS calculated for $\mathrm{gr} / \operatorname{Ir}(111)$. A non-uniform S-O coupling with a maximum strength of $\lambda_{\max }=0.5 t$ is assumed. The parameters correspond to $A_{x}=2 \lambda(y) / a t, A_{y}=0$ and we assume $A_{0}=0.06 \sqrt{ } 3 t(\approx 0.3 \mathrm{eV})$, where $t$ is the first-neighbour hopping parameter of graphene $(3 \mathrm{eV})$ and $a$ is the distance between carbon atoms.

of graphene, and exhibit a distribution of small islands, which could result in a spatial variation of the $\mathrm{S}-\mathrm{O}$ coupling, whose derivative, in turn, originates the pseudo-magnetic field able to confine electrons. Novel and more exotic effects can be expected if these features are combined with other properties of graphene, such as long-range magnetic order induced by molecular adsorption ${ }^{29}$, gauge fields induced by strains or the additional degrees of freedom found in twisted graphene bilayers and multilayers ${ }^{30}$.

Received 19 June 2014; accepted 30 October 2014; published online 15 December 2014

\section{References}

1. Laughlin, R. B. Quantized Hall conductivity in two dimensions. Phys. Rev. B 23, 5632-5633 (1981)

2. Hasan, M. Z. \& Kane, C. L. Topological insulators. Rev. Mod. Phys. 82, 3045-3067 (2010).

3. Qi, X-L. \& Zhang, S-C. Topological insulators and superconductivity. Rev. Mod. Phys. 83, 1057-1110 (2011).

4. Bernevig, B. A. \& Zhang, S-C. Quantum spin Hall effect. Phys. Rev. Lett. 96, 106401 (2006).

5. Tombros, N., Jozsa, C., Popinciuc, M., Jonkman, H. T. \& van Wees, B. J. Electronic spin transport and spin precession in single graphene layers at room temperature. Nature 448, 571-574 (2007).

6. Haldane, F. D. M. Model for a quantum Hall effect without Landau levels: Condensed-matter realization of the "Parity Anomaly". Phys. Rev. Lett. 61, 2015-2018 (1988).

7. Kane, C. L. \& Mele, E. J. Quantum spin Hall effect in graphene. Phys. Rev. Lett. 95, 226801 (2005).

8. Castro Neto, A. H., Guinea, F., Peres, N. M. R., Novoselov, K. S. \& Geim, A. K. The electronic properties of graphene. Rev. Mod. Phys. 81, 109-162 (2009).

9. Weeks, C., Hu, J., Alicea, J., Franz, M. \& Wu, R. Engineering a robust quantum spin Hall state in graphene via adatom deposition. Phys. Rev. X 1, 021001 (2011).

10. Guinea, F., Katsnelson, M. I. \& Geim, A. K. Energy gaps and a zero-field quantum Hall effect in graphene by strain engineering. Nature Phys. 6, 30-33 (2010).

11. Levy, N. et al. Strain-induced pseudo-magnetic fields greater than 300 Tesla in graphene nanobubbles. Science 329, 544-547 (2010).

12. Yan, W. Strain and curvature induced evolution of electronic band structure in twisted graphene bilayer. Nature Commun. 4, 2159 (2013).

13. N'Diaye, A. T., Coraux, J., Plasa, T. N., Busse, C. \& Michely, T. Structure of epitaxial graphene on $\operatorname{Ir}(111)$. New J. Phys. 10, 043033 (2008).

14. Barja, S. et al. Self-organization of electron acceptor molecules on graphene. Chem. Commun. 46, 8198-8200 (2010).

15. Busse, C. et al. Graphene on $\operatorname{Ir}(111)$ : Physisorption with chemical modulation. Phys. Rev. Lett. 107, 036101 (2011).

16. Sun, Z. et al. Topographic and electronic contrast of the graphene moiré on Ir(111) probed by STM and noncontact AFM. Phys. Rev. B 83, 081415(R) (2011).

17. Pletikosic, I. et al. Dirac cones and minigaps for graphene on $\operatorname{Ir}(111)$. Phys. Rev. Lett. 102, 056808 (2009).
18. Vlaic, S. et al. Cobalt intercalation at the graphene/Ir(111) interface: Influence of rotational domains, wrinkles and atomic steps. Appl. Phys. Lett. 104, 101602 (2014).

19. Petrovic, M. et al. The mechanism of Cs intercalation of graphene. Nature Commun. 4, 2772 (2013)

20. Miller, D. L. et al. Observing the quantization of zero mass carriers in graphene. Science 324, 924-927 (2009).

21. Katsnelson, M. I., Novoselov, K. S. \& Geim, A. K. Chiral tunneling and the Klein paradox in graphene. Nature Phys. 2, 620-625 (2006).

22. Katsnelson, M. I., Guinea, F. \& Vozmediano, M. A. H. Gauge fields in graphene. Phys. Rep. 496, 109-148 (2010).

23. Sun, J., Fertig, H. A. \& Brey, L. Effective magnetic fields in graphene superlattices. Phys. Rev. Lett. 105, 186501 (2010).

24. Gopalakrishnan, S., Ghaemi, P. \& Ryu, S. Non-Abelian SU(2) gauge fields through density wave order and strain in graphene. Phys. Rev. B 86, 081403 (2012).

25. San-Jose, P., Gonzalez, J. \& Guinea, F. Non-Abelian gauge potentials in graphene bilayers. Phys. Rev. Lett. 108, 216802 (2012).

26. Ma, D. \& Yang, Z. First principles studies of $\mathrm{Pb}$ doping in graphene: Stability, energy gap, and spin-orbit splitting. New J. Phys. 13, 123018 (2013).

27. Hunt, B. et al. Massive Dirac fermions and Hofstadter butterfly in a van der Waals heterostructure. Science 340, 1427-1430 (2013).

28. Woods, C. R. et al. Commensurate-incommensurate transition for graphene on hexagonal boron nitride. Preprint at http://arxiv.org/abs/1401.2637 (2014).

29. Garnica, M. et al. Long range magnetic order in a purely organic $2 \mathrm{D}$ layer adsorbed on epitaxial graphene. Nature Phys. 9, 368-374 (2013).

30. Geim, A. K. \& Grigorieva, I. V. van der Waals heterostructures. Nature 499, $419-425$ (2013)

\section{Acknowledgements}

We acknowledge support from the Spanish Ministry of Economy (MINECO) through Grant Nos FIS2010-18847, FIS2010-19609-C02-01, FIS2011-23713, FIS2012-33011, FIS2013-40667-P, Gobierno Vasco-UPV/EHU IT-756-13 and the European Research Council Advanced Grant (contract 290846), Comunidad de Madrid through grant MAD2D P2013/MIT-3007 and the European Commission under the Graphene Flagship, contract CNECT-ICT-604391. H.O. thanks the programme JAE-Pre (CSIC, Spain) for financial support.

\section{Author contributions}

The experiments were carried out primarily by F.C. and M.G., with important contributions from S.B., J.J.N. and A.B. The calculations were performed by H.O., M.M.O., E.V.C., A.A. and F.G. The data analysis was carried out by A.L.V.d.P. and F.C. The paper was written by R.M. and F.G. with contributions from F.C., A.L.V.d.P. and H.O

\section{Additional information}

Supplementary information is available in the online version of the paper. Reprints and permissions information is available online at www.nature.com/reprints.

Correspondence and requests for materials should be addressed to F.G. or R.M.

\section{Competing financial interests}

The authors declare no competing financial interests. 